Engineering and Computational Mechanics Volume 166 Issue EM1

Fibre-reinforced, self-compacting concrete flow modelled by smooth particle hydrodynamics Kulasegaram and Karihaloo

ice | proceedings
Proceedings of the Institution of Civil Engineers

Engineering and Computational Mechanics 166 March 2013 Issue EM1 Pages 22-31 http://dx.doi.org/10.1680/eacm.11.00004

Paper 1100004

Received 08/02/2011

Published online 27/07/2012

Keywords: computational mechanics/concrete structures/concrete

technology \& manufacture

ICE Publishing: All rights reserved

Accepted 23/05/2011
-

iae

Institution of Civil Engineers

publishing

\title{
Fibre-reinforced, self-compacting concrete flow modelled by smooth particle hydrodynamics
}

1 Sivakumar Kulasegaram BTech, MSc, PhD Lecturer, School of Engineering, Cardiff University, Cardiff, UK
2 Bhushan Lal Karihaloo MTech, PhD, DEng, DSc(hc), FICE, FIEAus, CPEng, MASME, FASCE

Laing O'Rourke Professor of Hybrid Concrete Innovation, School of Engineering, Cardiff University, Cardiff, UK
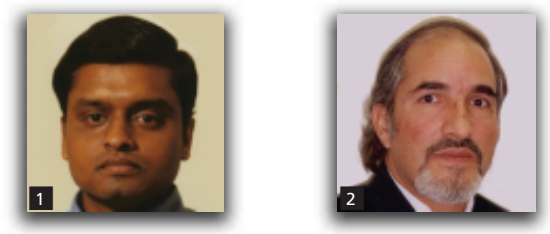

A Lagrangian particle-based method, the smooth particle hydrodynamics, is used to model the flow of ultra-highperformance, self-compacting concretes containing short steel fibres which behave like a non-Newtonian fluid described by a Bingham-type constitutive model. An incompressible smooth particle hydrodynamics method is used to simulate the flow after the kink in the shear stress against the shear strain rate constitutive equation is first appropriately smoothed out. One of the key factors that ensures the strength and durability of an ultra-highperformance concrete is the orientation of the fibres within the concrete structures cast from the ultra-high performance, self-compacting concretes. Therefore, this paper mainly focuses on developing a numerical methodology to determine how the fibres distribute and orient themselves during the ultra-high performance, selfcompacting concrete flow. For this, a novel approach which can be easily combined with the continuum flow model developed in a previous study by the authors is proposed here. A number of numerical simulations are presented to demonstrate the effectiveness of the proposed methodology.

\section{Notation}

d rate of deformation

g gravitational acceleration

$P \quad$ pressure

$t \quad$ time

$\boldsymbol{v}$ particle velocity

$\boldsymbol{x}$ particle position

$\dot{\gamma} \quad$ shear strain rate

$\eta \quad$ plastic viscosity

$\mu \quad$ effective plastic viscosity

$\rho \quad$ fluid density

$\tau \quad$ shear stress

$\tau_{0} \quad$ yield stress

\section{Introduction}

In recent years, self-compacting concrete (SCC) is increasingly replacing the conventional vibrated concrete in the construction industry. Moreover, the need for very high durability structures with a lower life cycle cost is promoting the use of ultra-highperformance, self-compacting concretes (UHPSCC) containing distributed short steel fibres. UHPSCC must exhibit excellent flow-ability in the formwork to ensure long-term durability. The filling behaviour of UHPSCC with short steel fibres is even more difficult to predict in formworks of complex shape in the presence of reinforcing steel. Therefore, in order to produce highquality UHPSCC structures it is vital to understand fully the flow characteristics of UHPSCC. The most cost-effective way to gain such an understanding is by performing numerical simulations. These will not only enable us to understand the filling behaviour, but will also provide an insight into how the fibres will distribute and orient themselves during the flow.

In practice, it is not easy to model the flow of UHPSCCs or any such heterogeneous materials due to their complex constitutive behaviour. Any computational model for the flow should be able to describe the rheological behaviour of the material accurately and to follow the large deformation and Lagrangian nature of the flow. A number of computational strategies have been attempted in the past to simulate the SCC flow by assuming the concrete to be a homogeneous viscous fluid and using either the discrete element method (Gram and Silfwerbrand, 2011; Noor and Uomoto, 1999; Petersson, 2003) or the Lagrangian finite-element method (Dufour and Pijaudier-Cabot, 2005). An overview of the various computational techniques used in the past to model 
concrete flow and their advantages and disadvantages is given in (Roussel et al., 2007). Due to the Lagrangian nature of the SCC flow and due to the fact that an SCC mix is essentially an aggregate of particles of different sizes and shapes, the use of particle-based Lagrangian numerical techniques to simulate such flows is both more appropriate and simpler than the traditional mesh-based methods (Dufour and Pijaudier-Cabot, 2005; Noor and Uomoto, 1999; Petersson, 2003; Roussel et al., 2007). Therefore, a Lagrangian particle-based technique, the so-called smooth particle hydrodynamics (SPH) method (Bonet and Kulasegaram, 2000; Monaghan, 1992, 1994) was chosen by authors (Kulasegaram et al., 2011) for simulating the SCC flow. As fibre orientations during the flow of an UHPSCC containing randomly distributed short steel fibres play an important role in determining the durability and strength of the hardened structures, this paper focuses on developing a methodology to predict the fibre orientations during the flow. A simple and robust numerical approach that can be easily combined with the SPH technique developed by the authors previously (Kulasegaram et al., 2011) is proposed here to determine the fibre orientations during the simulation of UHPSCC flow.

The simplicity and Lagrangian nature of SPH have been exploited in the past to model many free-surface fluid flows and related engineering problems (Bonet and Kulasegaram, 2000; Cummins and Rudman, 1999; Kulasegaram et al., 2004; Monaghan, 1992, 1994). To simulate the flow of a UHPSCC with short steel fibres, an incompressible SPH methodology was adopted in the present study. In Section 2, for the sake of completeness, the governing equations and basic formulation relating to incompressible SPH and the procedure for coupling the SPH with a suitable Bingham model to represent the rheological behaviour of UHPSCC are briefly reviewed (for full details, see Kulasegaram et al. (2011)). A novel methodology to determine fibre orientations during the flow is also detailed in this section. Section 3 presents numerical results for UHPSCC flow to demonstrate the effectiveness of the proposed methodology in determining the fibre orientations during the flow. Section 4 concludes the paper by highlighting the advantages of the presented numerical approach.

\section{Numerical modelling}

This section introduces the basic governing equations and numerical procedures associated with the modelling of UHPSCC flow.

\subsection{Governing equations}

The ultra-high-performance, self-compacting, fibre-reinforced concrete is assumed to have the characteristics of a viscous nonNewtonian fluid, described by a bi-linear Bingham-type rheological model in which the fluid flow only initiates once the shear stress has reached a critical value called the yield stress $\tau_{0}$ (Ghanbari and Karihaloo, 2009; Papanastasiou, 1987). Thereafter, the shear stress varies linearly with the shear strain rate $\dot{\gamma}$, the slope being equal to the plastic viscosity $\eta$ of the UHPSCC mix. Ghanbari and Karihaloo (2009) have shown how to predict the plastic viscosity $\eta$ of self-compacting concretes with and without short steel fibres from the measured viscosity of the paste alone using micromechanical models in which the second phase aggregates are treated as rigid spheres and the short steel fibres as slender rigid bodies. They have also argued that the yield stress $\tau_{0}$ of SCC mixes is practically unchanged over a very large range of plastic viscosities. From a computational point of view, it is expedient to approximate the bi-linear Bingham constitutive relation which has a kink at $\dot{\gamma}=0$ by a smooth function with a continuous first derivative; for example, by a scalar function of the type (Papanastasiou, 1987):

1. $\tau=\eta \dot{\gamma}+\tau_{0}\left(1-\mathrm{e}^{-m \dot{\gamma}}\right)$

in which $m$ is a very large number. Experience shows (Ghanbari and Karihaloo, 2009) that when $m>10^{5}$ is chosen the smooth function (1) is practically indistinguishable from the original bilinear constitutive relation.

The isothermal Lagrangian form of mass and momentum conservation equations are

2. $\frac{1}{\rho} \frac{\mathrm{D} \rho}{\mathrm{D} t}+\nabla \cdot v=0$

3. $\frac{\mathrm{D} \boldsymbol{v}}{\mathrm{D} t}=-\frac{1}{\rho} \nabla P+\boldsymbol{g}+\frac{1}{\rho} \nabla \cdot \boldsymbol{\tau}$

In Equations 2 and $3, \rho, t, \boldsymbol{v}, P, \boldsymbol{g}$ and $\boldsymbol{\tau}$ represent the fluid particle density, time, particle velocity, pressure, gravitational acceleration and shear stress, respectively. These equations govern both Newtonian and non-Newtonian fluid flows. However, the non-Newtonian fluids differ from Newtonian ones due to their shear rate-dependent viscosity. The shear stress in a non-Newtonian fluid is generally given by

$$
\text { 4. } \tau=2 \mu(\dot{\gamma}) \boldsymbol{d} \equiv\left(\eta+\frac{\tau_{0}}{\dot{\gamma}}\left(1-\mathrm{e}^{-m \dot{\gamma}}\right)\right) \boldsymbol{d} \quad(\dot{\gamma}>0)
$$

where $\boldsymbol{d}$ is the rate of deformation and $\mu$ is the effective plastic viscosity which is a function of shear rate. For the modelling of non-Newtonian SCC flow, the shear stress can be directly computed from Equation 1.

\subsection{Numerical solution procedure}

To track the Lagrangian non-Newtonian flow, a projection method based on a predictor-corrector time stepping scheme (Chorin, 1968; Cummins and Rudman, 1999; Koshizuka et al., 1998) is adopted here to solve the governing equations. 
The prediction step is an explicit integration in time without enforcing incompressibility. Only the viscous stress and gravity terms in Equation 3 are considered and an intermediate particle velocity $\boldsymbol{v}_{n+1}^{*}$ is obtained as

5. $\boldsymbol{v}_{n+1}^{*}=\boldsymbol{v}_{n}+\left(\boldsymbol{g}+\frac{1}{\rho} \nabla \cdot \boldsymbol{\tau}\right) \Delta t$

Then the correction step is executed by considering the pressure term in Equation 3

6. $\frac{\boldsymbol{v}_{n+1}-\boldsymbol{v}_{n+1}^{*}}{\Delta t}=-\left(\frac{1}{\rho} \nabla P_{n+1}\right)$

where $\boldsymbol{v}_{n+1}$ is the corrected particle velocity at the time level $n+1$.

The intermediate velocity $\boldsymbol{v}_{n+1}$ is usually not divergence-free but this is imposed on the corrected velocity $\boldsymbol{v}_{n+1}$ by enforcing the incompressibility condition from Equation 2

$$
\text { 7. } \nabla \cdot v_{n+1}=0
$$

Hence the intermediate velocity can be projected on the divergence-free space by writing the divergence of Equation 6 as

8. $\quad \nabla \cdot\left(\frac{1}{\rho} \nabla P_{n+1}\right)=\frac{\nabla \cdot v_{n+1}^{*}}{\Delta t}$

Since the density of a particle remains constant in the present simulation, Equation 8 can be rewritten as

9. $\quad \nabla^{2} P_{n+1}=\frac{\rho}{\Delta t} \nabla \cdot v_{n+1}^{*}$

where $\nabla^{2}$ is the Laplacian operator.

Once the pressure is obtained from Equation 9, the particle velocity is updated by the computed pressure gradient (see Equation 6)

10. $v_{n+1}=v_{n+1}^{*}-\left(\frac{1}{\rho} \nabla P_{n+1}\right) \Delta t$

Finally, the instantaneous particle position is updated using the corrected velocity

11. $\boldsymbol{x}_{n+1}=\boldsymbol{x}_{n}+\boldsymbol{v}_{n+1} \Delta t$
The time step $\Delta t$ is chosen based on the relevant Courant stability conditions for the given problem. In the case of a Bingham-type SCC fluid flow, the time step is primarily controlled by the plastic viscosity $\eta$. This is due to the fact that the maximum velocity of the flow is mainly affected by the viscosity of the flow. Therefore, the time step size is generally decided by

12. $\Delta t=\min \left(\frac{\alpha_{1} r_{0}}{V_{\max }}, \frac{\alpha_{2} r_{0}^{2} \rho}{\mu}\right)$

where $r_{0}$ is the initial particle spacing, $V_{\max }$ is the maximum particle velocity, and $\alpha_{1}$ and $\alpha_{2}$ are coefficients usually in the order of $0 \cdot 1$. These coefficients depend on the choice of SPH kernel functions and the nature of the engineering application.

\subsection{Procedure for modelling fibre orientation}

It is possible to develop a complicated and computationally very expensive technique to model the fibre distribution and orientations during the flow. For example, numerical techniques such as the discrete element method (Gram and Silfwerbrand, 2011) or the lattice Boltzmann technique (Svec et al., 2011) can be used to model the SCC fluid flow with fibre suspensions. However, the aim of this paper was to produce a simple and reliable methodology that does not deviate significantly from the original formulation and techniques developed in Kulasegaram et al. (2011).

In a previous preliminary investigation (Kulasegaram et al., 2010), the mass of each fibre was concentrated at its centre of mass, and the rigid body translation of this 'fibre' particle was monitored during the flow by way of its velocity vector. This gave an indication of how the randomly distributed fibres translate as rigid particles during the flow but cannot capture their rotations as rigid slender rods (Figure 1).

In order to be able to monitor both the rigid body translation and the rigid body rotation of the steel fibres during the flow, let us consider the flow of UHPSCC with short steel fibres at a given time in space. Figure 2 schematically shows the positions of SPH particles representing the fluid (the SCC mix) and fibres at a given time in space.

As fibre orientation is mainly dictated by the flow of SCC mix rather than the mass of the fibres, it is feasible to assume that the positions of the ends of a fibre are largely controlled by the fluid particles surrounding them. Accordingly, the entire domain is discretised into two types of particles: one for representing the fluid and the other for fibres. The initial positions of the pair of particles representing a fibre are generated randomly but with a constant distance (equal to the fibre length) between them. In addition, the particles representing the fibre ends are not allowed to overlap with the fluid particles. Each pair of particles representing a fibre is tagged throughout the simulation. 


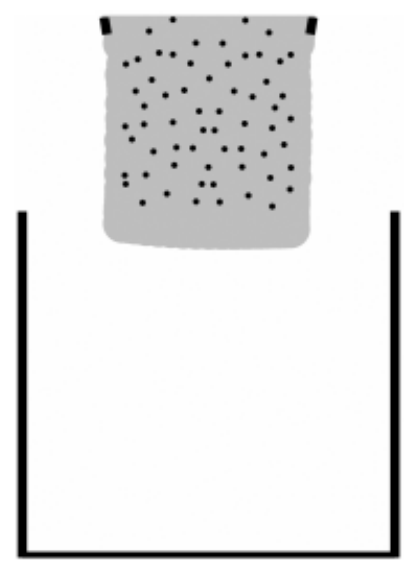

$0.15 \mathrm{~s}$

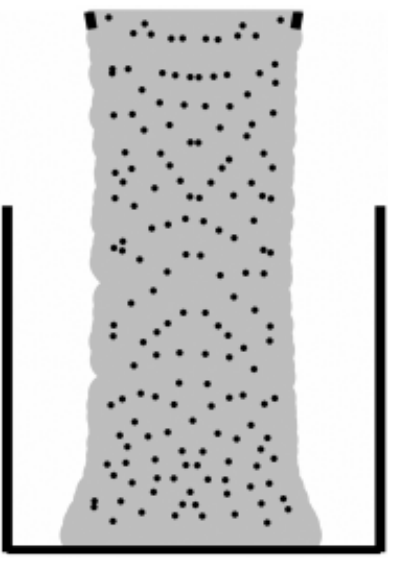

$0.25 \mathrm{~s}$

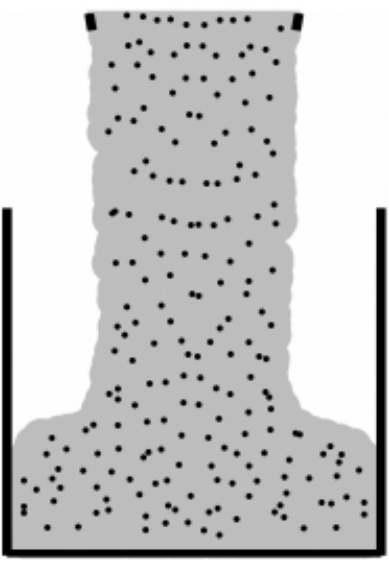

$0.30 \mathrm{~s}$

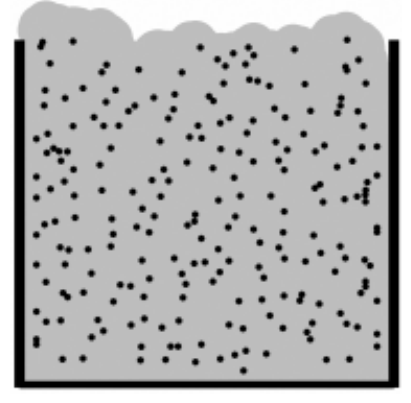

$0.59 s$

Figure 1. Filling of a mould with a square cross-section by UHPSCC. The outlet opening of the feed hopper is $100 \mathrm{~mm}$ above the rim of the mould. Dark dots represent randomly distributed rigid fibre 'particles'

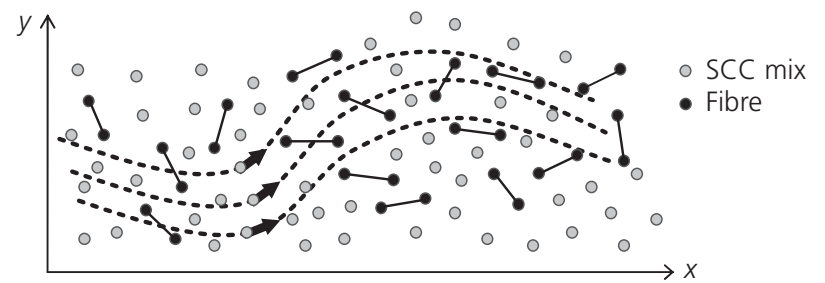

Figure 2. Schematic diagram of the flow of UHPSCC with rigid steel fibres

Both the fluid and fibre particles have the same continuum properties except that the masses of particles: representing the fibres are different from those of the fluid particles. In contrast to Figure 1 in which the fibre is treated as a particle with its mass concentrated at its centre, the mass of each fibre is equally divided between the particles at the ends of this fibre. Further, as the fibres are assumed to behave like rigid slender rods which undergo only rigid body translation and rotation during the flow, the fibre end particles are assumed to be rigidly connected. This condition is enforced by constraining the distance between the fibre end particles to remain unchanged (and equal to the physical length of the fibre) throughout the flow simulation.

This is done by implementing an additional step in the numerical procedure described in the previous section, as follows. It is noted first that following the computation of the pressure from Equation 9, the velocity and hence the particle positions are calculated from Equations 10 and 11, respectively. Now consider a single fibre between time steps $t_{n}$ and $t_{n+1}$ during which it has translated and rotated as a rigid body in the flow. Figure 3 illustrates the configuration of a fibre at time steps $t_{n}$ and $t_{n+1}$.

The distance between particle positions ${ }^{1} \boldsymbol{X}_{n+1}$ and ${ }^{2} \boldsymbol{X}_{n+1}$ at $t_{n+1}$

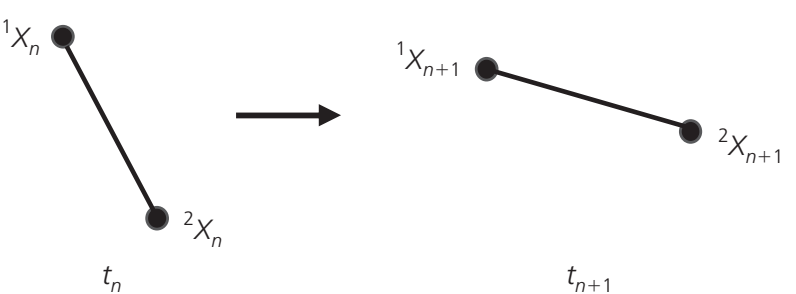

Figure 3. Schematic sketch of fibre orientations at $t_{n}$ and $t_{n+1}$

has to be equal to the fibre length. Therefore an iterative procedure has been introduced in computing the new positions of fibre end particles to ensure that they are separated by the fibre length. This iterative procedure has been combined with the steps given by Equations 10 and 11 to estimate the new positions of the particles. The key steps involved in enforcing the constant distance between the pair of particles representing a fibre can be summarised as follows, once the particle positions are updated using Equation 11 from time step $t_{n}$ to $t_{n+1}$.

(a) Step 1: check whether the distance $\left(\boldsymbol{L}_{n+1}\right)$ between the pair of particles representing each and every fibre is approximately equal to the fibre length $\left(\boldsymbol{L}_{0}\right)$.

(b) Step 2: if $\left|\boldsymbol{L}_{n+1}-\boldsymbol{L}_{0}\right|>10^{-5}$ for a certain fibre then equal and opposite penalty forces $F_{\mathrm{p}}$ proportional to $\left|\boldsymbol{L}_{n+1}-\boldsymbol{L}_{0}\right|$ are applied (in Equation 5) on the pair of particles representing that fibre along the vector connecting this pair of particles.

(c) Step 3: the Equations 5, 6, 9, 10 and 11 are now solved again sequentially to determine the positions of all the particles.

(d) Step 4: steps 1 to 3 are repeated until the pairs of particles representing all the fibres satisfy the condition $\left|\boldsymbol{L}_{n+1}-\boldsymbol{L}_{0}\right|<10^{-5}$. 
As discussed above, the rigid link between the pair of particles representing a fibre is enforced during the computations. Hence, any collisions between the fibres are simulated by way of interactions between the particles representing the individual fibres. As the fibre particles have the same continuum properties as the fluid particles, their interactions are modelled in the same manner as the interactions between the fluid particles.

The SPH formulation of the governing equations described in the previous section and the modelling of the boundary conditions follow the procedures described in Kulasegaram et al. (2011) and the references cited therein.

Based on the formulations detailed above, a numerical scheme which integrates the Lagrangian SPH formulations with the rheological Bingham model for UHPSCC has been developed. This scheme has been used to simulate how the UHPSCC fills a mould and how the randomly distributed fibres change their positions and orientations during this filling process. In the following section, a number of numerical examples are presented to validate the proposed numerical procedures.

\section{Numerical simulations}

In this section, two-dimensional numerical simulations of the UHPSCC flow in two different moulds were carried out. First, a rectangular mould with a square cross-section $(200 \mathrm{~mm} \times$ $200 \mathrm{~mm}$ ) was filled by UHPSCC containing $2 \cdot 5 \%$ by volume of randomly distributed $30 \mathrm{~mm}$ long rigid steel fibres. The UHPSCC mix was in a hopper whose outlet end was at a height of $50 \mathrm{~mm}$ above the top edge of the mould. To compare the effect of the plastic viscosity of the mix on the filling behaviour and fibre orientations, two different mix compositions were used in the simulation. The key difference in the model parameters between the two compositions was the plastic viscosity which changed with the amount of super-plasticiser used in the mix. The second mould also had a square cross-section but contained a circular cylindrical cut out along its length. In both the examples, realistic boundary conditions were assumed as in Kulasegaram et al. (2011), involving slip and friction between the UHPSCC and the contacting surfaces (e.g. the mould walls and the cut out surface).

\subsection{Filling of a rectangular mould with a square cross- section}

Figure 4 shows the hopper and the mould at time $t=0$. The hopper holds the UHPSCC mix containing $2.5 \%$ by volume of randomly distributed $30 \mathrm{~mm}$ long rigid steel fibres sufficient to fill the mould with a square cross-section, as shown in the figure. To investigate the effect of the plastic viscosity of the mix on the fibre distribution and orientations, two mix compositions UHPSCC 1 and UHPSCC2, differing only by the plastic viscosity, were considered. The rheological properties of these two mixes are given in Table 1.

Figures 5 and 6 show the filling of the mould by the two mixes at various times during the filling process. To view the fibre

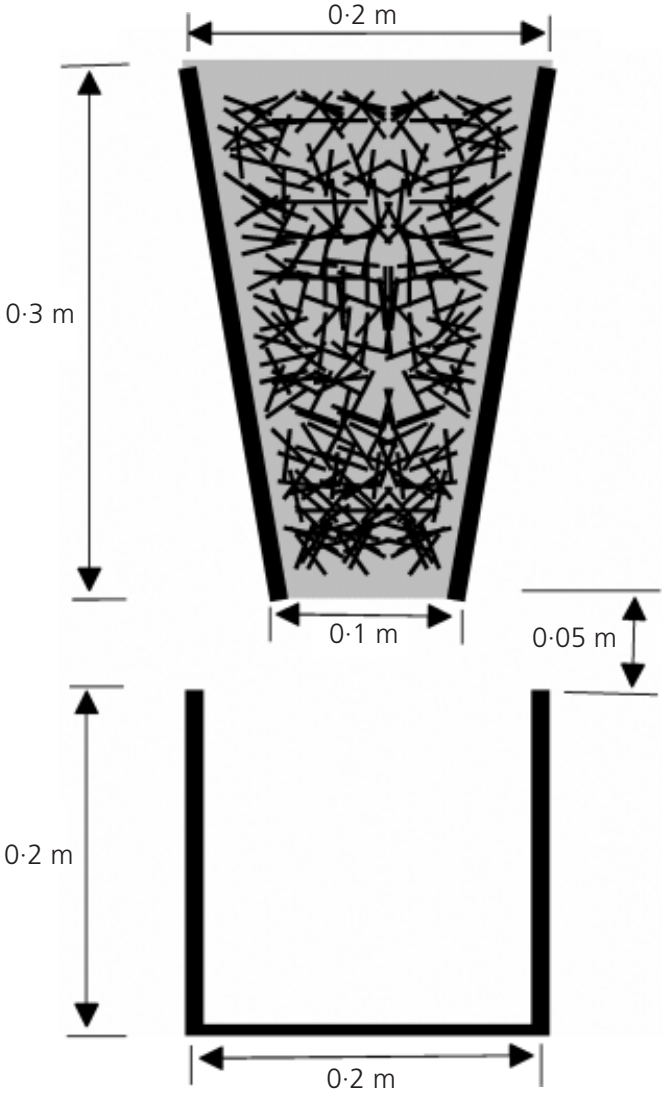

Figure 4. The hopper filled with UHPSCC and the empty mould at $t=0$

\begin{tabular}{lcc}
\hline Mix properties & UHPSCC1 & UHPSCC2 \\
\hline Density: $\mathrm{kg} / \mathrm{m}^{3}$ & 2411 & 2411 \\
Plastic viscosity: Pas & 70 & $54 \cdot 4$ \\
Yield stress: Pa & 200 & 200
\end{tabular}

Table 1. Density and rheological properties of UHPSCC1 and UHPSCC2

orientations clearly, only the flow when the mix was discharged from the hopper is illustrated in both Figures 5 and 6. It can be observed that in both mixes the flow pushed the fibres more towards the walls of the mould than to its centre. This was more noticeable in the mix with the lower plastic viscosity, namely UHPSCC2.

To investigate the influence of the gap between the hopper outlet and the top edge of the mould on the filling behaviour, the numerical simulations described above were repeated with a gap of $100 \mathrm{~mm}$. Figures 7 and 8 show the mould filling simulation by the two mixes at various stages of the filling process. It can be observed from these results that the vertical gap between the 
Engineering and Computational Mechanics Volume 166 Issue EM1
Fibre-reinforced, self-compacting concrete

flow modelled by smooth particle

hydrodynamics

Kulasegaram and Karihaloo

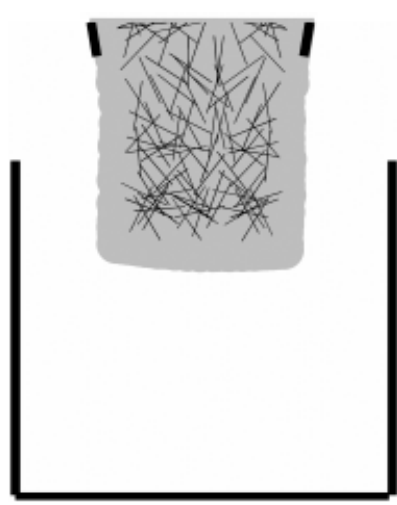

$0.15 \mathrm{~s}$

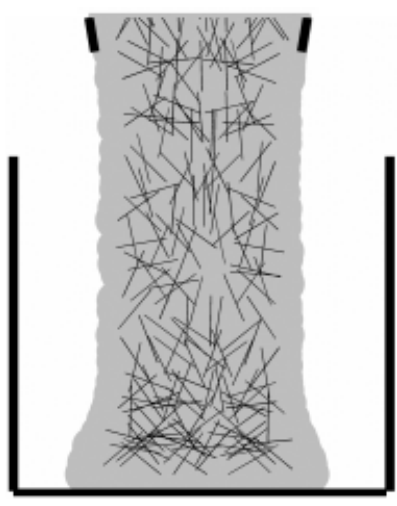

$0.23 \mathrm{~s}$

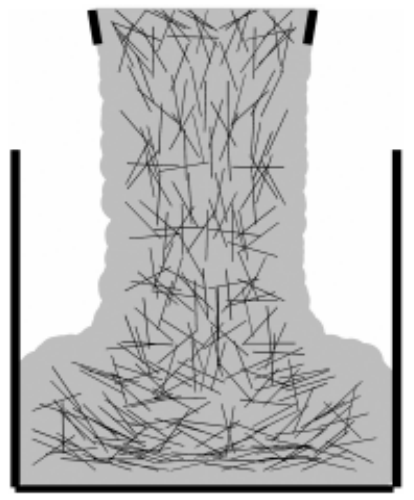

$0.28 \mathrm{~s}$
I

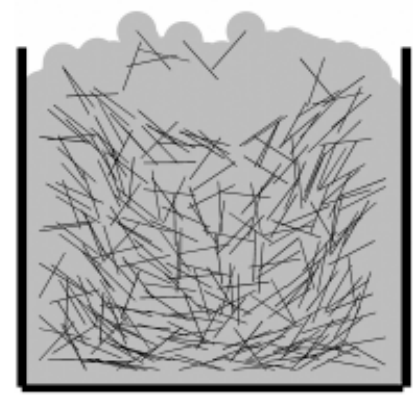

$0.56 \mathrm{~s}$

Figure 5. The hopper empties its contents of UHPSCC1 into the mould until the latter is filled. The outlet opening of the hopper is $50 \mathrm{~mm}$ above the rim of the mould

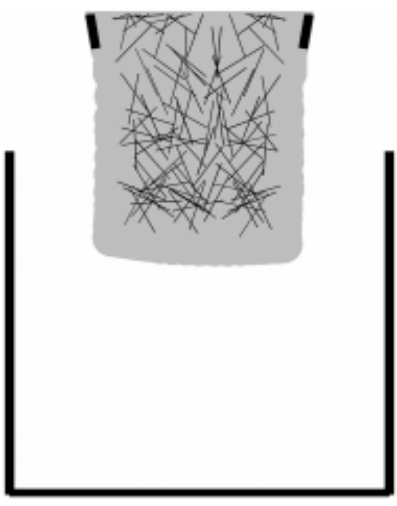

$0.15 \mathrm{~s}$

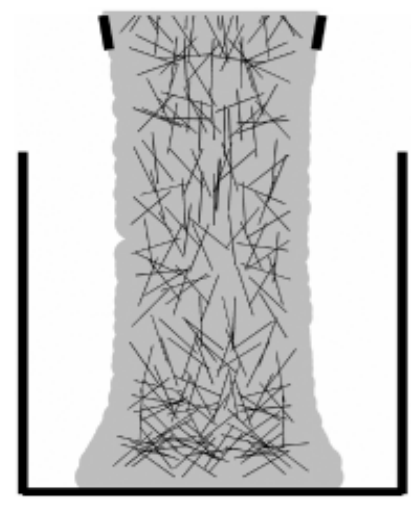

$0.23 \mathrm{~s}$

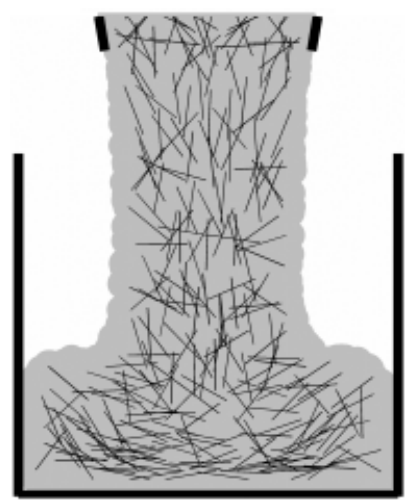

$0.28 \mathrm{~s}$
I I

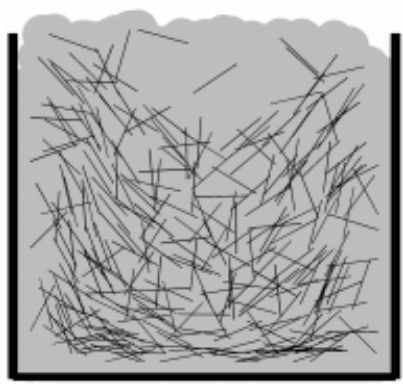

$0.54 \mathrm{~s}$

Figure 6. The hopper empties its contents of UHPSCC2 into the mould until the latter is filled. The outlet opening of the hopper is $50 \mathrm{~mm}$ above the rim of the mould

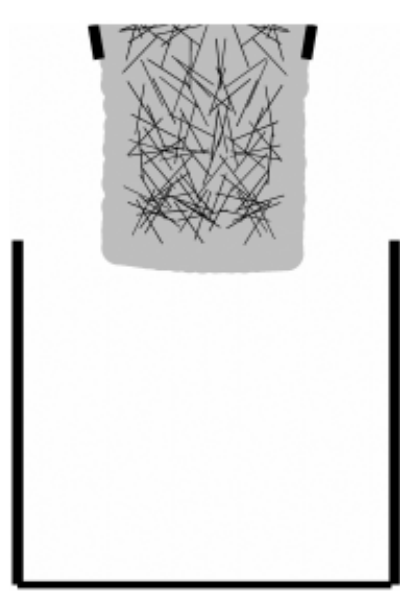

$0.15 \mathrm{~s}$

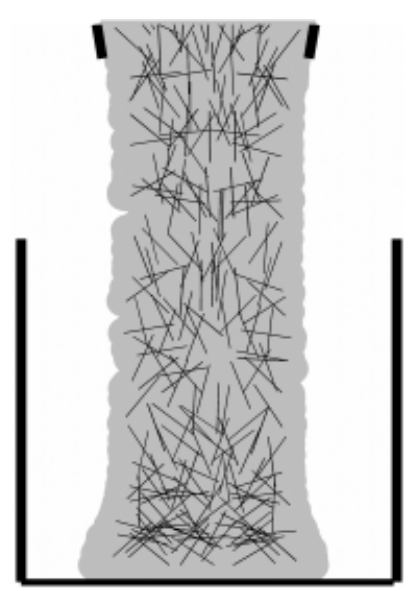

$0.25 \mathrm{~s}$

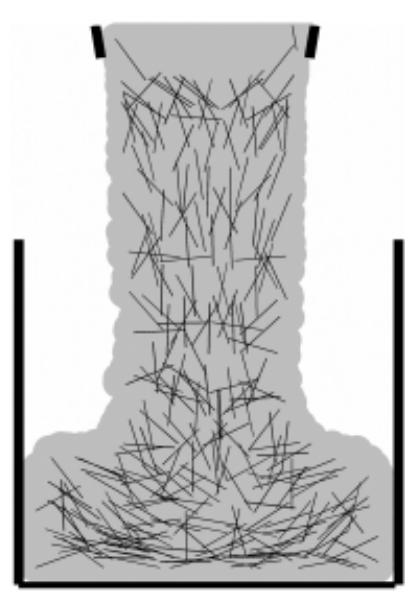

$0 \cdot 30 \mathrm{~s}$
I
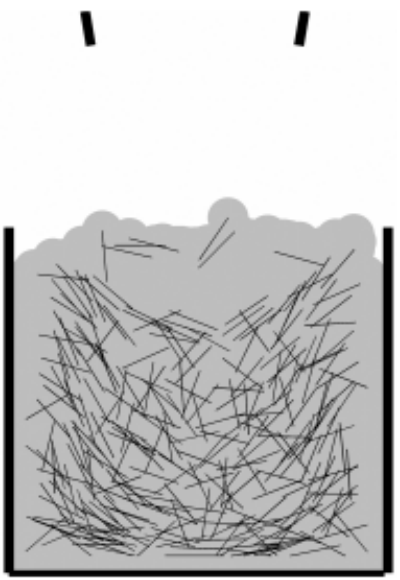

$0.58 \mathrm{~s}$

Figure 7. The hopper empties its contents of UHPSCC 1 into the mould until the latter is filled. The outlet opening of the hopper is $100 \mathrm{~mm}$ above the rim of the mould 
Engineering and Computational Mechanics Volume 166 Issue EM1
Fibre-reinforced, self-compacting concrete

flow modelled by smooth particle

hydrodynamics

Kulasegaram and Karihaloo

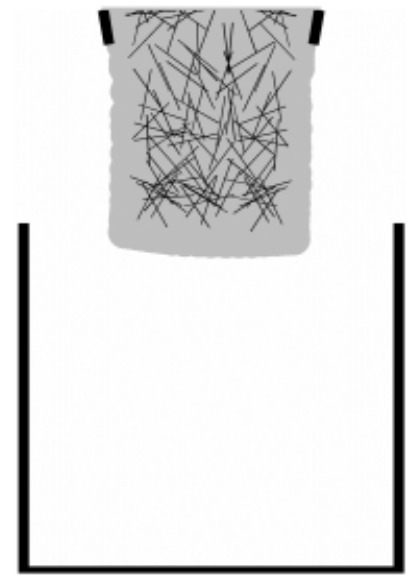

$0 \cdot 15 \mathrm{~s}$

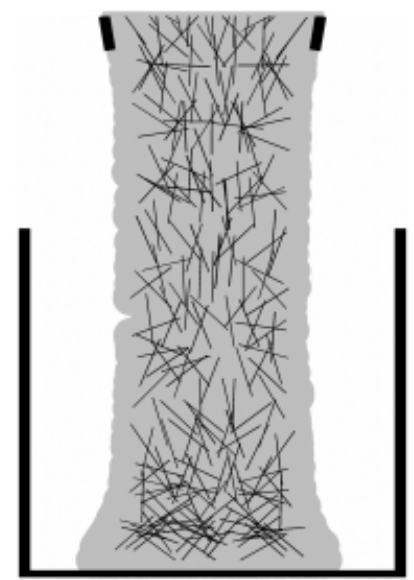

$0.25 \mathrm{~s}$

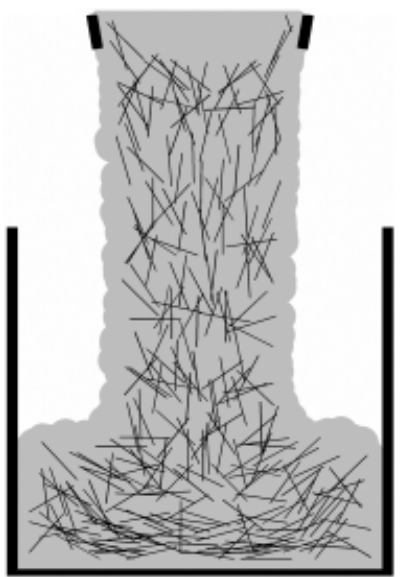

$0.30 \mathrm{~s}$
I

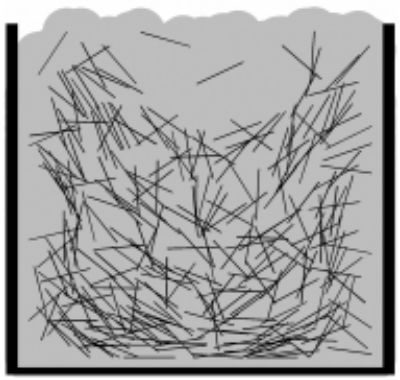

$0.56 \mathrm{~s}$

Figure 8. The hopper empties its contents of UHPSCC2 into the mould until the latter is filled. The outlet opening of the hopper is $100 \mathrm{~mm}$ above the rim of the mould

hopper outlet and the top edge of the mould did not significantly affect the flow behaviour of both mixes.

Furthermore, to study the effect of the hopper outlet opening on the flow behaviour the above example was repeated for both mixes with the outlet opening reduced by $50 \%$. Figures 9 and 10 illustrate the mould filling simulation by the two mixes at various stages of the filling process. It can be observed from a comparison of Figures 9 and 10 with Figures 7 and 8, respectively that a smaller outlet opening not only increased the filling time, as expected, but also tended to push the fibres more towards the walls of the mould.

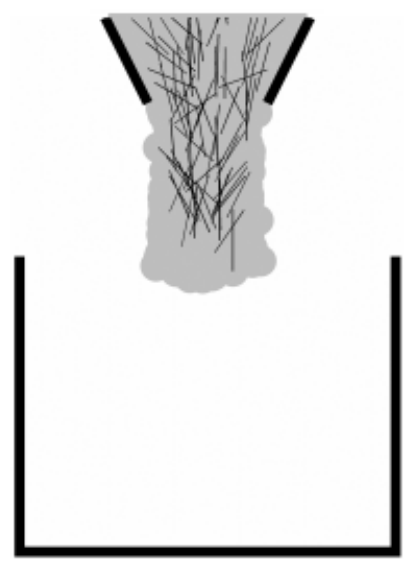

$0 \cdot 16 s$

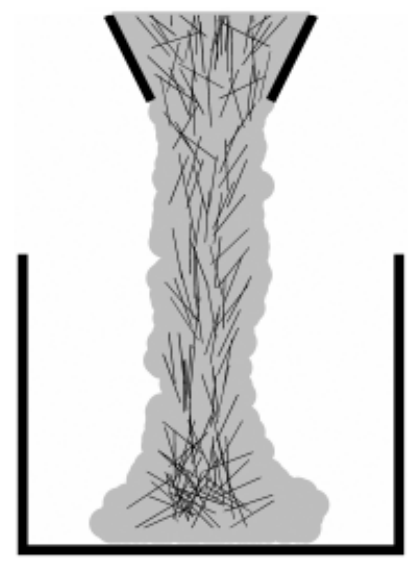

$0.26 \mathrm{~s}$

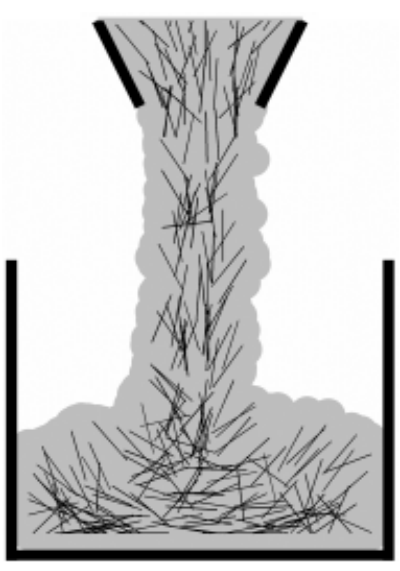

$0.42 \mathrm{~s}$

\subsection{Filling of a rectangular mould with a square cross-} section and a circular cylindrical obstruction

Consider now a second mould which also has a square crosssection but which contains a circular cylindrical cut out (obstruction) along its length. Such cut outs are built into the concrete beam elements during their casting and provide access for service ducting, and so on. Figure 11 shows the hopper and the mould cross-section with a circular obstruction. It was $90 \mathrm{~mm}$ in diameter and was located at the centre of the square section $(200 \mathrm{~mm} \times 200 \mathrm{~mm})$. Figures 12 (UHPSCC1) and 13 (UHPSCC2) show the mould with the obstruction being filled at various stages in the filling process and how the fibres were

Figure 9. The hopper with a narrower outlet opening empties its contents of UHPSCC 1 into the mould until the latter is filled. The outlet opening of the hopper is $100 \mathrm{~mm}$ above the rim of the mould 
Engineering and Computational Mechanics Volume 166 Issue EM1
Fibre-reinforced, self-compacting concrete

flow modelled by smooth particle

hydrodynamics

Kulasegaram and Karihaloo

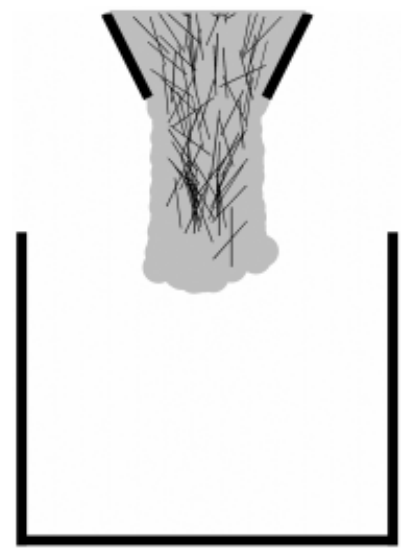

$0 \cdot 16 \mathrm{~s}$

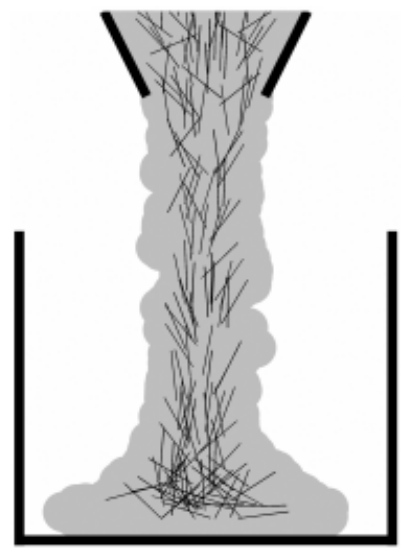

$0 \cdot 26 s$

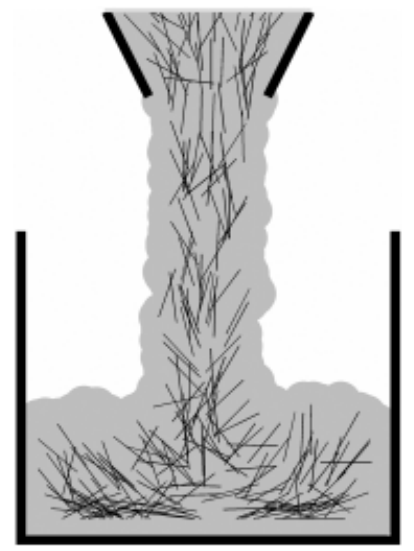

$0.40 \mathrm{~s}$
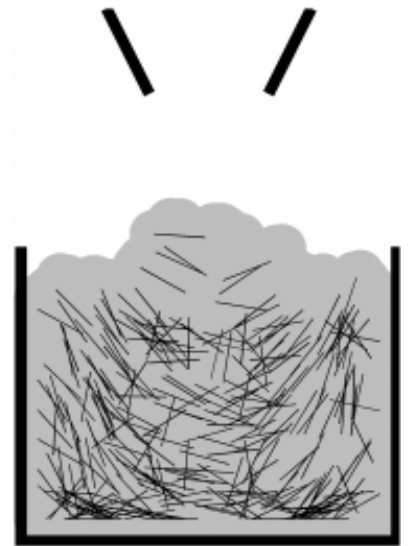

$0.80 \mathrm{~s}$

Figure 10. The hopper with a narrower outlet opening empties its contents of UHPSCC2 into the mould until the latter is filled. The outlet opening of the hopper is $100 \mathrm{~mm}$ above the rim of the mould

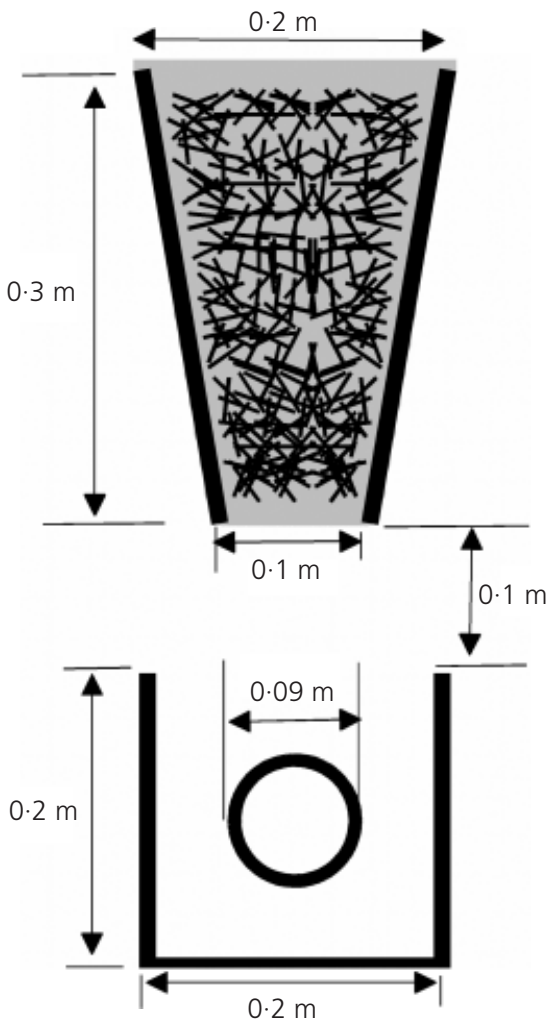

Figure 11. The hopper filled with UHPSCC and the empty mould with a circular obstruction at $t=0$

oriented. It appears that the fibres were concentrated along the surface of the circular obstruction.

In all the examples above, the volume of UHPSCC in the initial configuration was constant as shown in Figures 4 and 11. In the previous study (Kulasegaram et al., 2011) it was found that for the convergence of the solution it was sufficient to discretise the volume of the material (within the hopper) approximately by 4000 particles. Therefore, in all the above numerical simulations the initial volume was represented by 4000 particles. This number of particles was also sufficient to ensure that the smoothing length used in the SPH computations was much smaller than the fibre length, so that the pair of particles representing a fibre could not be direct neighbours of each other. In all the numerical simulations the time step was determined by Equation 12 and it was found to be in the order of $1.0 \times 10^{-6} \mathrm{~s}$.

In the SPH method, the implementation of the boundary conditions is not as straightforward as in the traditional mesh-based methods. In the present study, a technique based on rigid boundary particles with arrays of dummy particles was used to implement the wall boundary conditions, as described in Kulasegaram et al. (2011). As a result of the treatment of the boundary walls in this manner it can be noted from the results of the simulations that the fibres appeared not to touch the walls.

In all the above simulations the number of fibres was deliberately exaggerated to demonstrate the capability of the numerical procedure. For the considered volume, the number of fibre particles in the section in proportion to the total number of particles (i.e. 4000) can be estimated using the geometric probability theory. This number is much smaller than that used in the simulations.

\section{Conclusion}

A numerical methodology has been developed to predict how the fibres distribute and orient themselves during the filling of moulds with UHPSCC mixes that contain $2.5 \%$ of $30 \mathrm{~mm}$ long steel fibres which are initially randomly distributed. This method- 
Engineering and Computational Mechanics Volume 166 Issue EM1
Fibre-reinforced, self-compacting concrete

flow modelled by smooth particle

hydrodynamics

Kulasegaram and Karihaloo

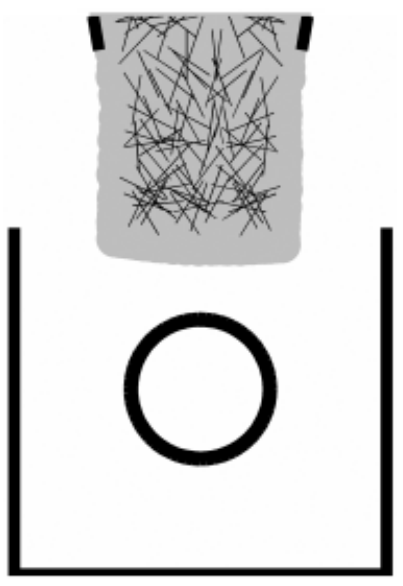

$0 \cdot 15 \mathrm{~s}$

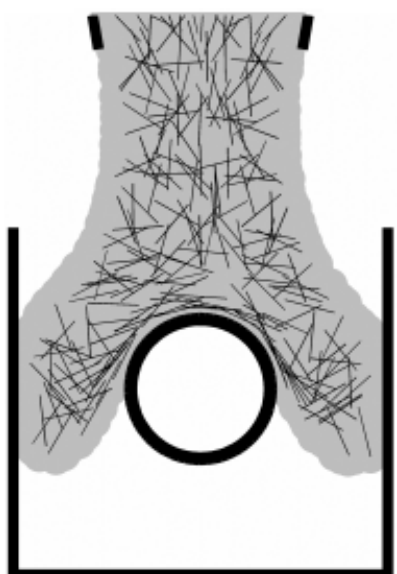

$0.25 \mathrm{~s}$

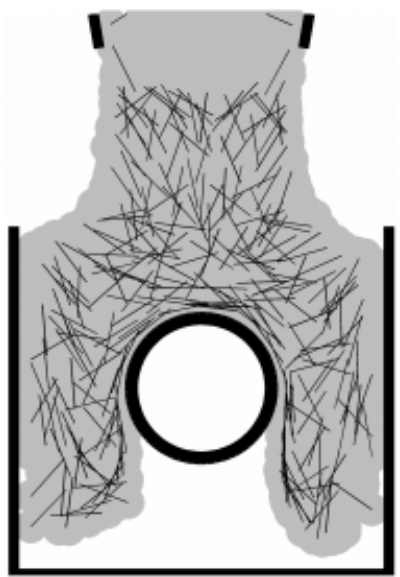

$0.31 \mathrm{~s}$
I

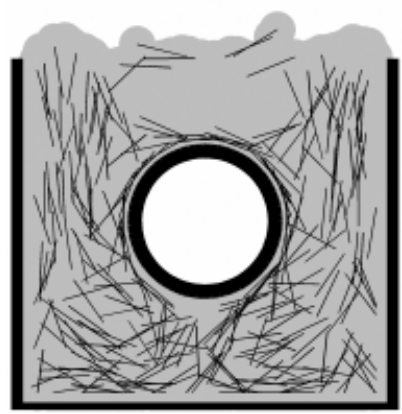

$0.50 \mathrm{~s}$

Figure 12. The hopper empties its contents of UHPSCC 1 into the mould with a circular obstruction until the mould is filled. The outlet opening of the hopper is $100 \mathrm{~mm}$ above the rim of the mould

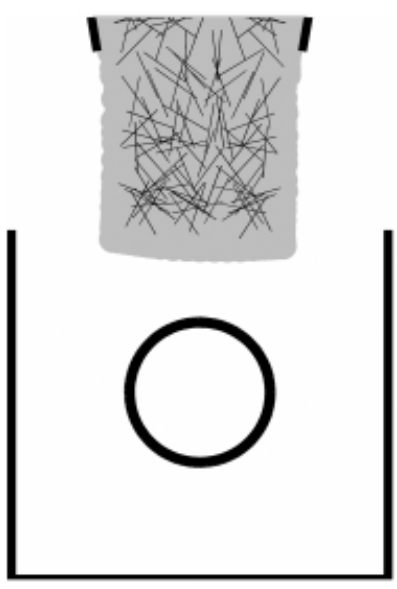

$0.15 s$

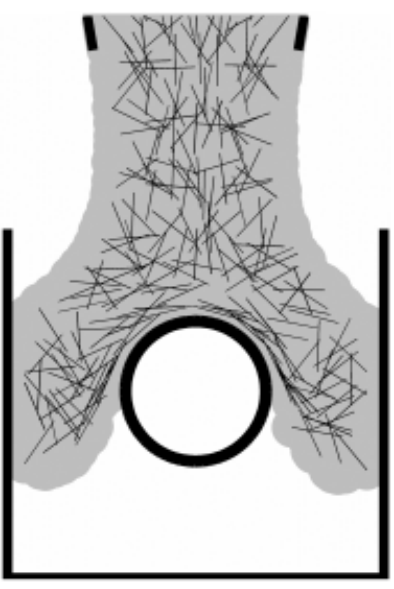

$0.25 \mathrm{~s}$

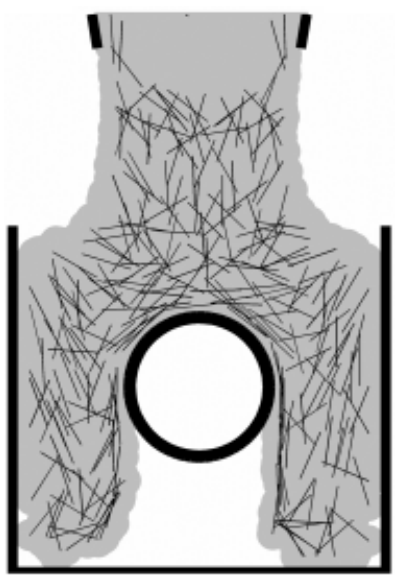

$0.31 \mathrm{~s}$
I

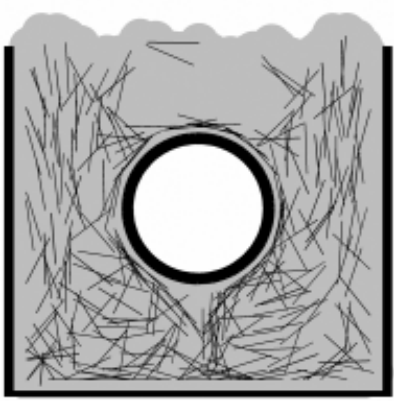

$0.47 \mathrm{~s}$

Figure 13. The hopper empties its contents of UHPSCC2 into the mould with a circular obstruction until the mould is filled. The outlet opening of the hopper is $100 \mathrm{~mm}$ above the rim of the mould

ology combines the corrected Lagrangian SPH method detailed in Kulasegaram et al. (2011) to simulate the flow of UHPSCC mixes with a procedure to exhibit the fibre orientations during the flow. Two examples confirmed that the developed SPH methodology was capable of predicting the behaviour of UHPSCC mixes during their flow in moulds. They showed clearly how the fibre distribution and orientation were influenced by the plastic viscosity of the mix. In particular, they revealed that the fibres tended to concentrate near the mould walls and the surfaces of any internal obstructions within the mould. The methodology developed in the present study can be used to investigate how this fibre concentration near the slipping and frictional walls and surfaces can be minimised by adjusting the mix rheological parameters and/or reducing the friction by greasing the surfaces with which the mix comes into contact during the flow. These and other aspects of mould filling are currently being investigated. Finally, it should be mentioned that a two-dimensional representation is somewhat misleading because it shows all the fibres ( $2.5 \%$ by volume) in a single plane. To obtain an accurate picture of the fibre distribution and orientation during the flow, a 
three-dimensional flow simulation is necessary. The present methodology can easily be extended to three dimensions. That work is also in progress.

\section{REFERENCES}

Bonet J and Kulasegaram S (2000) Correction and stabilization of smooth particle hydrodynamics methods with applications in metal forming simulation. International Journal for Numerical Methods in Engineering 47(6): 1189-1214.

Chorin AJ (1968) Numeical solution of the Navier-Stokes equations. Mathematics of Computation 22(104): 745-762.

Cummins SJ and Rudman M (1999) An SPH projection method. Journal of Computational Physics 152(2): 584-607.

Dufour F and Pijaudier-Cabot G (2005) Numerical modelling of concrete flow: homogeneous approach. International Journal for Numerical and Analytical Methods in Geomechanics 29(4): 395-416.

Ghanbari A and Karihaloo BL (2009) Prediction of the plastic viscosity of self-compacting steel fibre reinforced concrete. Cement and Concrete Research 39(12): 1209-1216.

Gram A and Silfwerbrand J (2011) Numerical simulation of fresh SCC flow: applications. Materials and Structures 44(4): 805 813.

Koshizuka S, Nobe A and Oka Y (1998) Numerical analysis of breaking waves using moving particle semi-implicit method. International Journal for Numerical Methods in Fluids 26(7): 751-769.

Kulasegaram S, Bonet J, Lewis RW and Profit MA (2004) Variational formulation based contact algorithm for rigid boundaries in two-dimensional SPH applications. Computational Mechanics 33(4): 316-325.

Kulasegaram S, Karihaloo BL and Ghanbari A (2010) Modelling the flow of self-compacting fibre reinforced concrete. Proceedings of the 5th SPHERIC Workshop, Manchester, UK, pp. 69-75.

Kulasegaram S, Karihaloo BL and Ghanbari A (2011) Modelling the flow of self-compacting concrete. International Journal for Numerical and Analytical Methods in Geomechanics 35(6): $713-723$.

Monaghan JJ (1992) Smoothed particle hydrodynamics. Annual Review of Astronomy and Astrophysics 30: 543574.

Monaghan JJ (1994) Simulating free surface flows with SPH. Journal of Computational Physics 110(2): 399-406.

Noor MA and Uomoto T (1999) Three-dimensional discrete element simulation of rheology tests of self-compacting concrete. In Proceedings of the 1st International RILEM Symposium on Self-Compacting Concrete. RILEM, Cachan, France, pp. 35-46.

Papanastasiou TC (1987) Flows of materials with yield. Journal of Rheology 31(5): 385-404.

Petersson Ö (2003) Simulation of self-compacting concrete laboratory experiments and numerical modelling of testing methods, J ring and L-box tests. In Proceedings of the $3 \mathrm{rd}$ International RILEM Symposium, Pro 33. RILEM, Cachan, France, pp. 202-207.

Roussel N, Geiker MR, Dufour F, Thrane LN and Szabo P (2007) Computational modelling of concrete flow : general overview. Cement and Concrete Research 37(9): 1298-1307.

Svec O, Skocek J, Stang H, Olesen JF and Poulsen PN (2011) Fully coupled lattice Boltzmann simulation of fiber reinforced self compacting concrete flow. In Proceedings of the 'Computers Methods in Mechanics', Warsaw, Poland.

\section{WHAT DO YOU THINK?}

To discuss this paper, please email up to 500 words to the editor at journals@ice.org.uk. Your contribution will be forwarded to the author(s) for a reply and, if considered appropriate by the editorial panel, will be published as a discussion in a future issue of the journal.

Proceedings journals rely entirely on contributions sent in by civil engineering professionals, academics and students. Papers should be 2000-5000 words long (briefing papers should be 1000-2000 words long), with adequate illustrations and references. You can submit your paper online via www.icevirtuallibrary.com/content/journals, where you will also find detailed author guidelines. 